\title{
Personalien
}

Todesfälle / Décès / Decessi

Carlo Albani (1947), † 17.12.2017,

Facharzt für Neurologie

8023 Zürich

Piero Valli (1978), † 25.12.2017,

Facharzt für Gastroenterologie und

Facharzt für Allgemeine Innere Medizin,

8006 Zürich

Roger Spiller (1938), † 18.12.2017,

Facharzt für Allgemeine Innere Medizin, 8038 Zürich

Günter Waldeck (1951), † 3.1.2018,

Facharzt für Allgemeine Innere Medizin,

8003 Zürich

Jürgen K. Willmann (1972), † 8.1.2018,

Facharzt für Radiologie,

CA 94305 Stanford, US

Frank Gafner (1921), † 26.1.2018,

Facharzt für Ophthalmologie,

4410 Liestal

Beat von Albertini (1944), † 29.1.2018,

Facharzt für Nephrologie,

1003 Lausanne

Katharina Burri-Bräm (1953), † 31.1.2018,

Fachärztin für Allgemeine Innere Medizin, 8580 Amriswil

Ljubisav Djordjevic (1930), † 6.2.2018,

4917 Busswil b. Melchnau
Praxiseröffnung /

Nouveaux cabinets médicaux /

Nuovi studi medici

AG

Scherwin Talimi,

Facharzt für Urologie. Cordulaplatz 6,

5400 Baden

VD

Michele Zanzi,

Spécialiste en chirurgie plastique,

reconstructive et esthétique, place Bel-Air 1, 1003 Lausanne

ZH

Andreas Schönbeck,

Facharzt für Allgemeine Innere Medizin Altstetterstrasse 118, 8048 Zürich

\section{Aargauischer Ärzteverband}

Zur Aufnahme in den Aargauischen Ärzteverband als ordentlich praktizierende Mitglieder haben sich angemeldet:

Elza Dimitrova, 8965 Berikon,

Fachärztin für Gynäkologie und Geburtshilfe, Praxiseröffnung in Praxisgemeinschaft in Berikon seit 5. Februar 2018

Thomas Ernst, 4312 Magden, Facharzt für Allgemeine Innere Medizin, FMH, Praxiseröffnung in Rheinfelden per 16. April 2018

Martin Ghedina, 8634 Hombrechtikon, Facharzt für Physikalische Medizin und Rehabillitation, FMH, angestellt in Praxis in Schafisheim seit 16. Januar 2018

Christine Heneka, 5073 Gipf-Oberfrick, Fachärztin für Allgemeine Innere Medizin, FMH, angestellt in Praxis in Sisseln seit 1. März 2018

Andrea Krümmer Käch, 6006 Luzern, Fachärztin für Gynäkologie und Geburtshilfe, angestellt in Praxis in Zofingen seit 1. März 2018

Afrim Kurtani, 4535 Hubersdorf, Facharzt für Chirurgie, Praxiseröffnung in Praxisgemeinschaft in Wohlen seit 1. Februar 2018

Eva Simon-Glaab, 5022 Rombach, Fachärztin für Allgemeine Innere Medizin, FMH, angestellt in Praxis in Aarau per 1. April 2018

Als Assistenz- und Oberarztmitglieder hat sich angemeldet:

Fabienne Renetseder, 5000 Aarau, Assistenzärztin im Kantonsspital Aarau seit 1. März 2017

Diese Kandidaturen werden in Anwendung von Art. 5 der Statuten des Aargauischen Ärzteverbandes veröffentlicht. Einsprachen müssen innert 14 Tagen seit der Bekanntmachung schriftlich und begründet der Geschäftsleitung des Aargauischen Ärzteverbandes eingereicht werden. Nach Ablauf der Einsprachefrist entscheidet die Geschäftsleitung über Gesuch und allfällige Einsprachen. 
Ärztegesellschaft des Kantons Bern

Ärztlicher Bezirksverein Bern Regio

Zur Aufnahme als ordentliches Mitglied hat sich angemeldet:

\section{Michael Glenck,}

Facharzt für Radiologie, Lindenhofspital, Bremgartenstrasse 117, 3012 Bern

Helmut Swart, Praktischer Arzt,

Sanacare Gruppenpraxis,

Bubenbergplatz 10, 3011 Bern

Zur Aufnahme als ordentliches Mitglied in unselbständiger Tätigkeit hat sich angemeldet:

\section{Till Schnittfeld,}

Facharzt für Kinder- und Jugendpsychiatrie und -psychotherapie,

Mühlemattstrasse 62, 3007 Bern

Einsprachen gegen diese Vorhaben müssen innerhalb 14 Tagen seit der Veröffentlichung schriftlich und begründet beim Präsidenten des Ärztlichen Bezirksvereins Bern Regio eingereicht werden. Nach Ablauf der Frist entscheidet der Vorstand über die Aufnahme der Gesuche und über die allfälligen Einsprachen.

Ärztegesellschaft des Kantons Schwyz Zur Aufnahme in die Ärztegesellschaft des Kantons Schwyz hat sich angemeldet:

Jens Busche,

Facharzt für Allgemeine Innere Medizin, FMH, plant Übernahme Praxis Dr. Renggli Küssnacht a.R. 02/2019

Jürg Gresser, Leitender Arzt Chirurgie Spital Einsiedeln, FMH

Debbie Rauchenstein-Kleinert, Fachärztin für Gynäkologie und Geburtshilfe, FMH, eröffnet ab Mai 2018 Kinderwunschzentrum Kopelli Klinik Pfäffikon SZ.

Einsprachen gegen diese Aufnahme richten Sie schriftlich innert 20 Tagen an Dr. med. Uta Kliesch, Maria-Hilf-Strasse 9, 6430 Schwyz oder per mail an uta.kliesch@hin.ch

\section{Ärztegesellschaft des Kantons Luzern}

Zur Aufnahme in unsere Gesellschaft Sektion Stadt hat sich gemeldet:

Katja Röllin Galliker, Fachärztin für Kinderund Jugendmedizin, FMH, ab 1. April 2018: Kinderarztpraxis am Bahnhof, Robert-ZündStrasse 2, 6005 Luzern

Renate Müller, Fachärztin für Kinder- und Jugendpsychiatrie, FMH, ab Mitte April 2018: Praxis für Kinder- und Jugendpsychiatrie, Maihofstrasse 52, 6004 Luzern

Einsprachen sind innert 20 Tagen nach der Publikation schriftlich und begründet zu richten an: Ärztegesellschaft des Kantons Luzern, Schwanenplatz 7, 6004 Luzern

\section{Ärztegesellschaft Thurgau}

Zum Eintritt in die Ärztegesellschaft Thurgau haben sich gemeldet:

Marion Buchwald, Konstanzerstrasse 45, 8280 Kreuzlingen,

Fachärztin für Kinder- und Jugendmedizin

Bernhard Jäschke, Imterstrasse 42, D-88326 Aulendorf, Praktischer Arzt

Andreas David Kistler Weber Seidenstrasse 17, 8400 Winterthur, Facharzt für Allgemeine Innere Medizin und Facharzt für Nephrologie, FMH

Gabriela Cristina Rossi Ezeanyika,

Freudenbergstrasse 11c, 9512 Rossrüti, Fachärztin für Allgemeine Innere Medizin, FMH
Unterwaldner Ärztegesellschaft

Zur Aufnahme in die Unterwaldner Ärztegesellschaft hat sich angemeldet:

Marcelo Sebastian Walker, Facharzt für Allgemeine Innere Medizin, FMH, ab 1.4.2018 Praxistätigkeit: Praxis Dr. med. Martin Sigg, Seestrasse 49, 6052 Hergiswil/NW

Einsprachen gegen diese Aufnahme sind mit Begründung innert 20 Tagen an die Präsidentin der Unterwaldner Ärztegesellschaft, Dagmar Becker, Mondmattli 3, 6375 Beckenried, zu richten.

Ärzte-Gesellschaft des Kantons Zug

Zur Aufnahme in die Ärzte-Gesellschaft des Kantons Zug als ordentliches Mitglied hat sich angemeldet:

Anne Tai Zweifel,

Fachärztin für Ophthalmologie, FMH, spez. Ophthalmochirurgie, Feldhof 7, $6300 \mathrm{Zug}$

Einsprachen gegen diese Kandidatur müssen innerhalb 14 Tagen seit dieser Veröffentlichung schriftlich und begründet beim Sekretariat der Ärzte-Gesellschaft des Kantons Zug eingereicht werden. Nach Ablauf der Einsprachefrist entscheidet der Vorstand über Gesuch und allfällige Einsprachen. 